\title{
Medical Image of the Week: Diffuse Gastric Bleeding and ALL
}
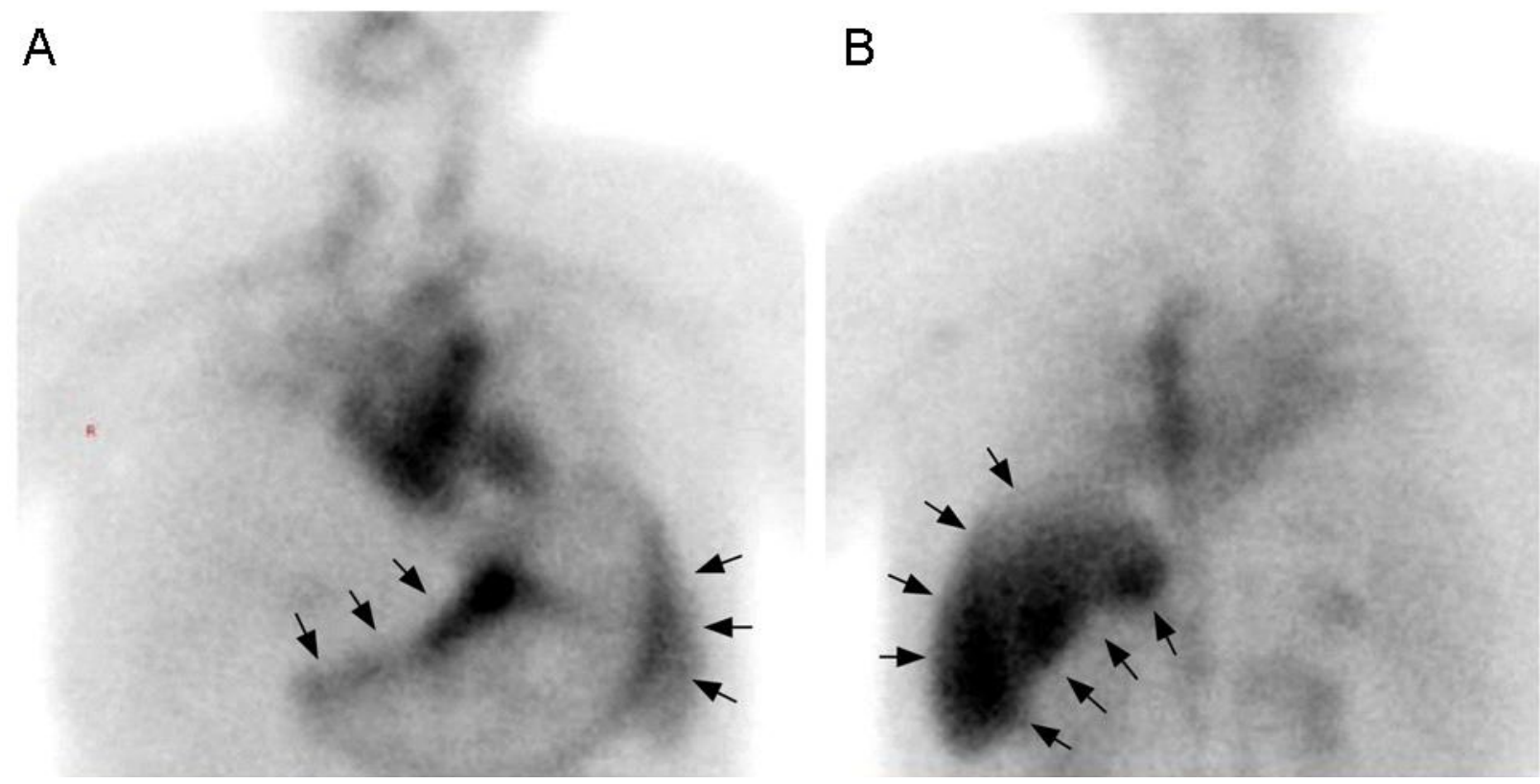

Figure 1. Technetium 99m tagged RBC scan showing abnormal radio tracer accumulation throughout the stomach (Panel $A$ ), and subsequently passing into the small bowel (Panel B).

A 26-year-old man with a medical history significant for acute lymphoblastic leukemia (ALL) presented with hypovolemic shock secondary to large volume hematemesis. The patient was diagnosed with ALL and treated with high dose chemotherapy followed by peripheral blood stem cell transplant from a matched unrelated donor one year prior to presentation. His treatment course was complicated by grade 4 acute graft versus host disease (GVHD) and CMV colitis. Blood work on admission showed hemoglobin of 6.4 $\mathrm{g} / \mathrm{dL}$ and a leukocytosis. Patient was intubated for airway protection, transferred to ICU, and EGD was performed, which revealed diffusely friable mucosa, inflammation, and ulcerations throughout the gastric mucosa with only a few areas of normal appearing mucosa. Additionally, areas of spontaneous bleeding were seen. Selective arteriography within the right gastric and gastroduodenal arteries showed no active extravasation from the stomach or duodenum. However the gastroepiploic and right gastric arteries were prophylactically embolized. Subsequently, a technetium 99m tagged RBC scan demonstrated abnormal radio-tracer accumulation throughout the stomach with subsequent passage into the small bowel (Figure 1). The patient continued to have refractory gastric bleeding even with an increased dose of cyclosporine. Surgical measures including gastrectomy were discussed with the family. However, the family decided on comfort care. The patient died the following day.

Although gastric bleeding is rare in ALL patients in general, it is more commonly associated with certain condition such as GVHD and colitis following allogeneic stem cell transplantation (SCT). One of the retrospective studies with 447 SCT patients 
showed that $21.1 \%$ of study population experienced major $\mathrm{GI}$ bleeding, requiring transfusions or surgical intervention. Also, their mortality was shown to be twice higher than patients without bleeding complication, although most cases of bleeding were mild and occurred in the peri-transplant period with concurrent severe thrombocytopenia (1).

Hemorrhagic complications occur predominantly during the first month of post transplant, and bleeding is more commonly associated with allogeneic SCT compared to autologous SCT (2). This is mainly secondary to GVHD with gastrointestinal involvement, which leads to destruction and fragility of the epithelium as well as hyperperfusion and proliferation of the blood vessels. As such, the risk of hemorrhage in patients with acute and chronic GVHD greater than grade I was 2.9 and 4.2 fold higher, respectively, and these patients had 10.8 fold higher risk of severe bleeding. The risk of bleeding is further increased by CMV infection, which infects vascular endothelial cells, narrows capillary lumens, and leads to ischemia and ulceration of gastric mucosa (3). The combination of GVHD and CMV infection could have synergistically damaged the gastric mucosa leading to severe refractory bleeding in our case.

Onyemaechi Okolo MD ${ }^{1}$, Seongseok Yun MD PhD ${ }^{1}$, Faiz Anwer MD, FACP

${ }^{1}$ Department of Medicine

${ }^{2}$ Department of Hematology \& Oncology, Blood \& Bone Marrow Transplantation Program

University of Arizona

Tucson, AZ, 85721

\section{References}

1. Pihusch R, Salat C, Schmidt E, Göhring P, Pihusch M, Hiller E, Holler E, Kolb HJ. Hemostatic complications in bone marrow transplantation: a retrospective analysis of 447 patients. Transplantation. 2002;74(9):1303-9. [CrossRef] [PubMed]

2. Törnebohm E, Lockner $D$, Paul $C$. A retrospective analysis of bleeding complications in 438 patients with acute leukaemia during the years 1972-1991. Eur J Haematol. 1993;50(3):160-7. [CrossRef] [PubMed]

3. Cheung AN, Ng IO. Cytomegalovirus infection of the gastrointestinal tract in nonAIDS patients. Am J Gastroenterol. 1993;88(11):1882-6. [PubMed] 